allow distal alveolar imaging at micron resolution in an ex vivo ovine model of bacterial infection.

Results AMP-1 demonstrates bacterial binding affinity in a concentration dependent manner and labels a diverse panel of bacteria, including a panel consisting of $>70 \%$ of ventilatorassociated pneumonia causing organisms and the pathogenic fungi Aspergillus fumigatus. AMP-1 demonstrates significantly higher fluorescence over isomolar linear equivalents for E. coli, K. pneumoniae, P. aeruginosa, MSSA, A. baumannii and S. pneumoniae (all $\mathrm{p}<0.01$ ), is selective for bacteria over mammalian cells and has improved chemical stability over the linear equivalent when incubated with bronchoaoveolar lavage from patients with acute respiratory distress syndrome. Furthermore, AMP-1 can label E. coli, K. pneumoniae, P. aeruginosa and MSSA in situ in an ex vivo ovine model when instilled endobronchially and imaged with FCFM (pin vitro and remains selective for gramnegative bacteria over mammalian cells. In the ex-vivo model AMP-2 selectively labels the gram-negative bacterial segments $(P$. aeruginosa, K. pneumonia and E. coli) over the gram-positive (MSSA, MRSA and S. pneumoniae) or control pulmonary segments (all $\mathrm{p}<0.05)$.

Conclusions A Smartprobe/FCFM strategy to immediately detect bacteria with gram selectivity in size relevant pre-clinical models is described, and are undergoing first-in-man translation.

\section{T5 MICRORNA-140-5P REGULATES DISEASE PHENOTYPE IN EXPERIMENTAL PULMONARY ARTERIAL HYPERTENSION VIA SMURF1}

${ }^{1}$ AMK Rothman, ${ }^{1} \mathrm{ND}$ Arnold, ${ }^{1} \mathrm{JA}$ Pickworth, ${ }^{1} \mathrm{~J}$ Iremonger, ${ }^{2} \mathrm{~L}$ Ciuclan, ${ }^{2} \mathrm{R}$ Allen, ${ }^{2} \mathrm{~S}$ GuthGundel, ${ }^{3} \mathrm{M}$ Southwood, ${ }^{3} \mathrm{NW}$ Morrell, ${ }^{1} \mathrm{SE}$ Francis, ${ }^{2} \mathrm{DJ}$ Rowlands, ${ }^{1} \mathrm{~A}$ Lawrie. ${ }^{1}$ University of Sheffield, Sheffield, UK; ${ }^{2}$ Novartis Institutes for Biomedical Research, Basel, Switzerland; ${ }^{3}$ University of Cambridge, Cambridge, UK

\subsection{6/thoraxjnl-2015-207770.5}

Introduction and objectives Clinical therapies for the treatment of pulmonary arterial hypertension (PAH) target vasoconstriction. However, the proliferative pulmonary vascular remodelling that drives disease persists contributing to significant patient morbidity and mortality. MicroRNA (miR) are short non-coding RNA that mediate post-transcriptional regulation of mRNA targets. We hypothesise that dysregulation of miR leads to derepression of cellular targets central to disease pathogenesis. We sought to identify dysregulated circulating miR in patients with $\mathrm{PAH}$, determine their phenotypic effect using in vitro and in vivo models and identify key mechanistic regulators that may represent novel therapeutic targets.

Methods Two patient cohorts were used to identify and validate differential expression of $\mathrm{miR}$ in whole blood by microarray and single assay $\mathrm{qPCR}$. Binding site and network analysis was used to identify key miR targets. Effect of miR on identified targets and disease phenotype was determined in pulmonary artery smooth muscle cells (PASMC) and in the monocrotaline (MCT) and Sugen 5416 plus Hypoxia (SuHx) models of PAH.

Results Expression of miR-140-5p was reduced in whole blood samples from patients with $\mathrm{PAH}$ and experimental models of $\mathrm{PAH}$. Network and pathway analysis identified key regulators of TGFß and PDGF signalling as miR-140-5p targets. Transfection with miR-140-5p inhibitor resulted in increased proliferation and migration of PASMC and de-repression of key targets. Nebulised delivery of miR-140-5p mimic prevented the development of PAH in the MCT rat model and attenuated progression of established $\mathrm{PAH}$ in MCT and SuHx rat models. In experimental models levels of SMURF1 protein correlated inversely with miR$140-5 \mathrm{p}$. Direct regulation of SMURF1 by miR-140-5p was demonstrated in vitro by 3'UTR luciferase activity. Both miR-140-5p mimic and SMURF1 siRNA increased BMP response element activity identifying SMURF1 as a key negative regulator of BMP signalling in PASMC. Genetic ablation of SMURF1 in C57BL6 mice conferred allele dependent protection from $\mathrm{SuHx}$ induced PAH. Finally, whole blood mRNA and pulmonary vascular immunoreactivity of SMURF1 was increased in patients with PAH.

Conclusions These studies suggest that miR-140-5p and SMURF1 are key regulators of BMP signalling and disease pathology in PAH and highlight SMURF1 as a potential novel therapeutic target.

\section{T6 MET TARGETED THERAPY IN LUNG ADENOCARCINOMA: DOES 'RESISTANT' EGFR MAKE A MET-RESPONSIVE DIMER?}

${ }^{1}$ RW Lee, ${ }^{1} \mathrm{E}$ Ortiz-Zapater, ${ }^{2} \mathrm{G}$ Weitsman, ${ }^{3} \mathrm{G}$ Fruhwirth, ${ }^{1} \mathrm{~W}$ Owen, ${ }^{2} \mathrm{~T} \mathrm{Ng},{ }^{1} \mathrm{G}$ Santis. ${ }^{1}$ Division of Asthma, Allergy and Lung Biology, Guy's Hospital, King's Health Partners, London, UK; ${ }^{2}$ Richard Dimbleby Department of Cancer Research, Randall Division of Cell and Molecular Biophysics, King's College London, London, UK; ${ }^{3}$ Department of Imaging Chemistry and Biology Division of Imaging Sciences and Biomedical Engineering School of Medicine, King's College London, London, UK

\subsection{6/thoraxjnl-2015-207770.6}

Introduction Lung cancer has extremely poor survival with few effective treatments. Activating EGFR mutations (e.g. L858R), select for impressive EGFR tyrosine kinase inhibitor (TKI) responses but most develop resistance e.g. T790M mutations or MET amplification, which is thought to mediate EGFR-HER3 kinase switching. Preclinical studies suggest EGFR-MET TKI synergy and whilst phase III trials have failed, optimism remains for biomarker driven therapy.

Hypothesis EGFR-MET dimerisation determines MET TKI response.

Objectives

1. Explore MET TKI responsiveness in EGFR mutant lung adenocarcinoma.

2. Develop an EGFR-MET FLIM assay indicative of MET TKI responsiveness.

Methods An in vitro and murine xenograft model was derived from EGFR TKI resistant NCI-H1975 lung adenocarcinoma cells (L858R T790M). After shEGFR knockdown we re-expressed EGFR L858R or WT to represent three clinical scenarios: 1. H1975: 'EGFR-TKI Resistant' 2. H1975 L858R: 'EGFR Responders' or 3. H1975 WT (wildtype EGFR).

Cells and xenografts (gavage) were challenged with exquisitely selective MET TKI, SGX523. Response was assessed by BrdU proliferation assays in vitro alongside random migration assays mimicking tumour cell motility. Xenograft tumours (FFPE) were stained for Ki67/phosphohistoneH3 (proliferation) and Masson's trichrome/anti-sma (Collagen/stroma).

EGFR-MET interaction was assessed by co-immunoprecipitation and Forster resonance energy transfer (FRET) FLIM to quantify EGFR-MET dimers after SGX523 treatment.

Results SGX523 treatment significantly reduced H1975 xenograft tumour growth/weight. Proliferation was suppressed in vitro (BrdU \%) and in vivo (phosphohistone-H3). Conversely in H1975 L858R, SGX523 reduced stroma (Masson's trichrome) and migration. EGFR-MET dimers were more common in H1975 than H1975 L858R/WT. This was associated with a 
mesenchymal, migratory phenotype in the H1975 cells whilst H1975 L858R were more proliferative.

SGX523 reduced EGFR-MET dimerization (FRET \%) in H1975 cells/FFPE xenograft tumours but induced dimer formation in H1975 L858R. H1975 WT showed little response to MET inhibition and low EGFR-MET FRET. In treated mice, FRET correlated with Ki67.

Conclusions

1. MET therapy was most effective in H1975 suggesting greater MET dependence with EGFR L858R/T790M.

2. EGFR L858R-T790M interacted most with Untreated MET.

3. MET inhibition reduced EGFR-MET dimerization in H1975 but increased H1975-L858R FRET.

4. EGFR-MET FRET assays can be applied to 'FFPE' processed lung cancer tissue from murine xenografts.

\section{FRET(\%) EGFR:MET Murine Xenograft}

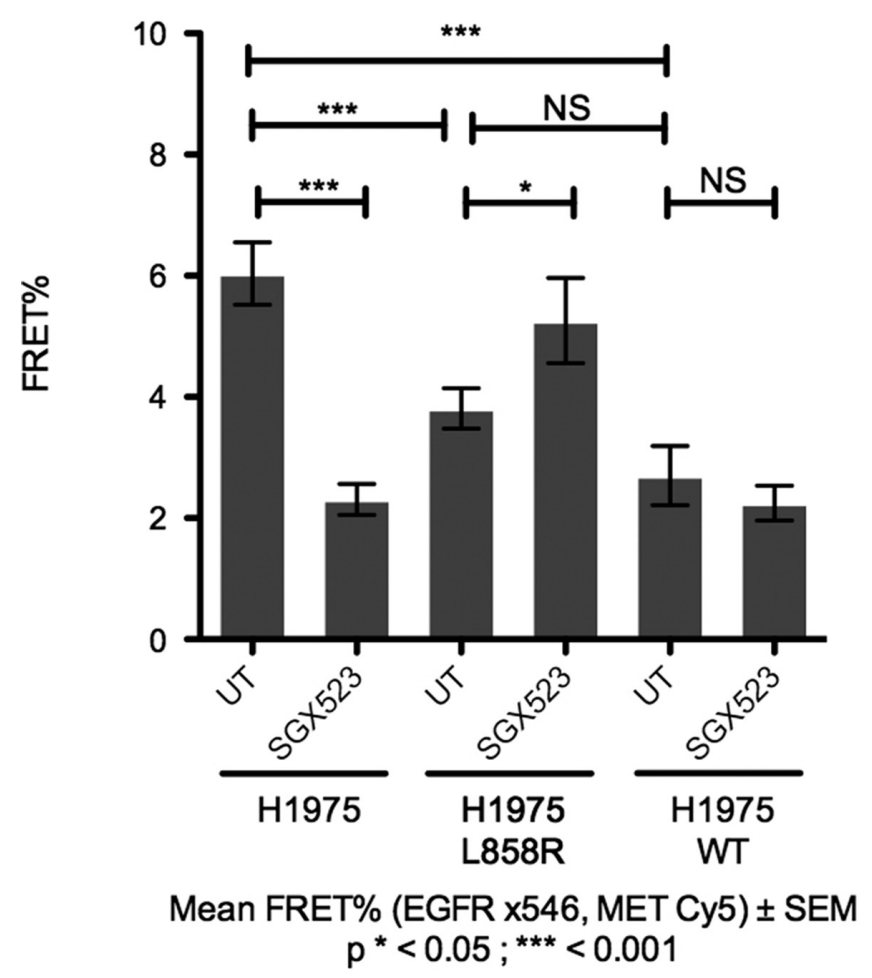

Abstract T6 Figure 1 


\section{Cutting edge pulmonary hypertension}

\section{S1* DOES PARADOXICAL EMBOLI OF PARTICULATE MATTER THROUGH PULMONARY ARTERIOVENOUS MALFORMATIONS PRECIPITATE MIGRAINES?}

${ }^{1} \mathrm{~T}$ Patel, ${ }^{2} \mathrm{~A}$ Elphick, ${ }^{3} \mathrm{JE}$ Jackson, ${ }^{4} \mathrm{CL}$ Shovlin. ${ }^{1}$ St George's, University of London and Imperial College School of Medicine, London, UK; ${ }^{2}$ Imperial College School of Life Sciences, London, UK; ${ }^{3}$ Imperial College Healthcare NHS Trust, London, UK; ${ }^{4}$ Imperial College London, London, UK

\subsection{6/thoraxjn1-2015-207770.7}

Introduction and objectives Pulmonary arteriovenous malformations (PAVMs) are an example of a right-to-left shunt resulting in deoxygenated, unfiltered venous blood bypassing the pulmonary capillaries to re-enter the systemic arterial circulation. Patients with PAVMs are known to have an increased incidence of migraines, reducing following PAVM treatment by embolisation. This study aimed to examine if paradoxical embolism of particulate matter through PAVMs may precipitate migraines.

Methods A structured survey was designed for online completion by people with hereditary haemorrhagic telangiectasia (HHT), the most common cause of PAVMs. Question logic directed participants through a series of unbiased questions that asked about HHT features including presence of PAVMs; variables in relation to migraines; and whether participants had undergone imaging tests. Stratified by whether contrast had been given, participants reporting migraines were asked whether any difference in migraines had been noted following scans, by ticking that "migraines were no different really"; "seemed a bit better"; "seemed a bit worse"; "seemed to bring on a migraine"; "seemed to stop a migraine". Participants were recruited from 26/07/2013- 21/04/2015, yielding 702 consented responses. Data were downloaded to an Excel spreadsheet for participant stratifications, and statistical analyses using GraphPad Prism 6.0 and STATA 13.1 (Statacorp LP).

Results Overall, 557 participants had HHT, of whom 180 $(32.3 \%)$ reported features consistent with migraines. HHT participants with migraines more commonly reported PAVMs than those without migraines $(62.8 \%$ vs $38.5 \%, \mathrm{p}<0.00001)$. For computerised tomography (CT) scans, images "with injection of contrast" were associated with a higher proportion of participants reporting worsening migraines than "without injection of contrast" CT scans $(11.7 \%$ vs $3.4 \%, \mathrm{p}=0.0065)$. This association strengthened following paired analysis of participants who had undergone both methods $(13.6 \%$ vs $3.2 \%, p=0.0032)$. In multiple regression analyses, there was no additional contribution from other participant demographics such as alcohol consumption or smoking habit. Analysis of magnetic resonance imaging (MRI), contrast echocardiography and ultrasound data is ongoing.

Conclusion This study strongly indicates that an association between injecting contrast media and the worsening of migraines, in participants with right-to-left shunts due to PAVMs, exists. Further research is required to establish the exact mechanism responsible for this phenomenon.

*S1- BTS Medical Student Award Highly Commended.
S2 VASCULAR QUIESCENCE FACTOR BMP9 IS REGULATED BY INFLAMMATION AND NEUTROPHIL ACTIVATION

W Li, L Long, K Hoenderdos, PD Upton, X Yang, AM Condliffe, ER Chilvers, NW Morrell. University of Cambridge, Cambridge, UK

\subsection{6/thoraxjnl-2015-207770.8}

Introduction Endothelial bone morphogenetic protein type II receptor (BMPR-II)-mediated signalling is essential for protecting vascular endothelium. Loss of BMPR-II predisposes human pulmonary artery endothelial cell (hPAEC) monolayers to apoptosis and increased permeability. In vivo, reduced BMPR-II function promotes endothelial permeability and the development of pulmonary arterial hypertension (PAH). Importantly, BMP9, the only confirmed active circulating BMP, signals preferentially via BMPRII and induces BMPR-II expression to maintain endothelial integrity and homeostasis. It was recently shown that administration of recombinant BMP9 prevented LPS-induced lung vascular leakage in vivo and reversed established $\mathrm{PAH}$ in three rodent models. ${ }^{1}$ However, it is not known how circulating BMP9 is regulated during LPS-induced inflammation and in PAH.

Objective To investigate whether BMP9 is regulated by inflammatory stimuli in vivo and in vitro.

Results Intraperitoneal LPS challenge in mice led to a significant increase in circulating neutrophil elastase levels with a reciprocal reduction in BMP9 levels (both measured by ELISA) within $24 \mathrm{~h}$. Since this reduction in BMP9 might be due to reduced BMP9 synthesis in the liver or cleavage of BMP9 by neutrophil-derived proteases, we quantified BMP9 synthesis in the liver after LPS challenge, as well as changes in alpha- 1 antitrypsin, the major elastase inhibitor in man. Synthesis of BMP9 fell sharply $3 \mathrm{~h}$ after LPS-challenge but recovered completely by $18 \mathrm{~h}$. No increase in the synthesis or levels of circulating active alpha- 1 antitrypsin was observed. Supernatants from purified human peripheral blood neutrophils activated in vitro degraded recombinant BMP9. Inhibition studies confirmed that the BMP9-cleavage activity released by activated neutrophils was largely attributable to neutrophil elastase.

Conclusions and discussions Synthesis of the endothelial protective factor BMP9 is actively regulated by inflammation, and BMP9 is subject to neutrophil elastase-mediated cleavage. Since inflammation has been shown to be a second hit in the pathogenesis of $\mathrm{PAH}$, this study could provide a potential link between inflammation and reduced endothelial BMPR-II signalling.

\section{REFERENCE}

1 Long L, Ormiston ML, Yang $X$, et al. Selective enhancement of endothelial BMPRII with BMP9 reverses pulmonary arterial hypertension. Nat. Med. 2015;21: $777-785$

\section{S3 1 REDUCED BMPR2 EXPRESSION POTENTIATES A PULMONARY ARTERY SMOOTH MUSCLE CELL SPECIFIC IL-1B RESPONSE}

1J Pickworth, ${ }^{2} S$ Shay, ${ }^{2} S$ Gladson, ' I Iremonger, 'AMK Rothman, 'S Francis, ${ }^{2} \mathrm{~J}$ West, ${ }^{1}$ A Lawrie. ${ }^{1}$ University of Sheffield, Sheffield, UK: ${ }^{2}$ Vanderbilt Instiute, Nashville, USA

\subsection{6/thoraxjnl-2015-207770.9}

Introduction and objectives Bone morphogenetic protein receptor type 2 (BMPR2) mutations are found in heritable and idiopathic pulmonary arterial hypertension however penetrance is incomplete implying necessity for a 'second hit'. IL-1ß and IL-6 are increased in $\mathrm{PAH}$ patients and animal models and are 